\title{
LETTERS
}

\section{Access to palliative care does not mean the quality of care was good}

In their article in CMAJ, Downar and colleagues ${ }^{1}$ provided a descriptive analysis of patient characteristics for patients who have received medical assistance in dying (MAiD) in comparison to patients who died from any cause.

One of the authors' stated intentions was to address the concern that patients may seek MAiD as a consequence of poor access to palliative care. Therefore, how access to palliative care was defined and captured in this study is of paramount importance to any associations implied.

In the absence of a clearly accepted and standardized definition for quality of palliative care delivery, it is critical that the definition used is at minimum systematic, transparent and reproducible. In this regard the authors have not provided their definition or objective criteria by which palliative care delivery was judged. The authors also do not provide an internal control to validate the approach used. They applied their definition for palliative care delivery only to patients who received MAiD, and not to a similar control-matched group of patients who died without MAiD.

If a very broad definition of access to palliative care were applied, then it would appear as though access to palliative care among patients who received MAiD was high - namely $75 \%$. However, this may not address the actual concern that patients receiving MAiD do not have access to adequate or meaningful palliative care provided by sufficiently qualified practitioners. This concern is substantiated by Dr. Downar's own words from a recent tweet: "We used the judgment of the RN investigators based on their review of the chart to arrive at $75 \%$. Indeed, that might refer to any type or quality of PC [palliative care]."2

Consequently, the observation of high prevalence of access to palliative care among patients may have been a foregone conclusion based on broad and subjectively applied criteria for palliative care involvement. It is especially critical that investigators in this domain focus on patient-important definitions for research, ideally with patient involvement from the outset. When one desires palliative care, one desires quality palliative care, not merely a construct called "access to palliative care." The use of surrogate constructs for key definitions in this way can be misleading to public discourse.

\section{Philippe D. Violette MSc MDCM}

Urologist; assistant professor, McMaster

University, Hamilton, Ont.

\section{Thomas P. Bouchard MD}

Family physician; clinical lecturer, University of Calgary, Calgary, Alta.

Cite as: CMAJ 2020 May 19;192:E558. doi: $10.1503 / \mathrm{cmaj} .74920$

\section{References}

1. Downar D, Fowler RA, Halko R, et al. Early experience with medical assistance in dying in Ontario, Canada: a cohort study. CMAJ 2020;192: E173-81.

2. Downar J. "We used the judgment of the RN investigators based on their review of the chart to arrive at $75 \%$. Indeed, that might refer to any type or quality of PC [palliative care]". Twitter 2020 Feb. 12. Available: https://twitter.com/jamesdownar/ status/1227777599348277248 (accessed 2020 Feb. 13).

Competing interests: None declared. 